\title{
Imbalance of arginine and asymmetric dimethylarginine is associated with markers of circulatory failure, organ failure and mortality in shock patients
}

\author{
Marlieke Visser ${ }^{1,2}$, Mechteld A. R. Vermeulen ${ }^{1}$, Milan C. Richir ${ }^{3}$, Tom Teerlink ${ }^{4}$, Alexander P. J. Houdijk ${ }^{3}$, \\ Piet J. Kostense ${ }^{5}$, Willem Wisselink ${ }^{1}$, Bas A. J. M. de Mol$^{2}$, Paul A. M. van Leeuwen ${ }^{1 *}$ and \\ Heleen M. Oudemans-van Straaten ${ }^{6}$ \\ ${ }^{1}$ Department of Surgery, VU University Medical Center, PO Box 7057, 1007 MB Amsterdam, The Netherlands \\ ${ }^{2}$ Department of Cardiothoracic Surgery, Academic Medical Center, University of Amsterdam, Amsterdam, The Netherlands \\ ${ }^{3}$ Department of Surgery, Medical Center Alkmaar, PO Box 501, 1800 AM Alkmaar, The Netherlands \\ ${ }^{4}$ Department of Clinical Chemistry, VU University Medical Center, PO Box 7057, 1007 MB Amsterdam, The Netherlands \\ ${ }^{5}$ Department of Epidemiology and Biostatistics, VU University Medical Center, PO Box 7057, 1007 MB Amsterdam, \\ The Netherlands \\ ${ }^{6}$ Intensive Care Unit, Onze Lieve Vrouwe Gasthuis, PO Box 95500, 1090 HM Amsterdam, The Netherlands
}

(Submitted 28 January 2011 - Final revision received 16 June 2011 - Accepted 20 July 2011 - First published online 1 December 2011)

\begin{abstract}
In shock, organ perfusion is of vital importance because organ oxygenation is at risk. NO, the main endothelial-derived vasodilator, is crucial for organ perfusion and coronary patency. The availability of NO might depend on the balance between a substrate (arginine) and an inhibitor (asymmetric dimethylarginine; ADMA) of NO synthase. Therefore, we investigated the relationship of arginine, ADMA and their ratio with circulatory markers, disease severity, organ failure and mortality in shock patients. In forty-four patients with shock (cardiogenic $n$ 17, septic $n$ 27), we prospectively measured plasma arginine and ADMA at intensive care unit admission, Acute Physiology and Chronic Health Evaluation (APACHE) II-(predicted mortality) and Sequential Organ Failure Assessment (SOFA) score, and circulatory markers to investigate their relationship. Arginine concentration was decreased (34.6 (SD 17.9) $\mu$ mol/1) while ADMA concentration was within the normal range $(0.46(\mathrm{SD} 0 \cdot 18) \mu \mathrm{mol} / \mathrm{l})$, resulting in a decrease in the arginine:ADMA ratio. The ratio correlated with several circulatory markers (cardiac index, disseminated intravascular coagulation, bicarbonate, lactate and pH), APACHE II and SOFA score, creatine kinase and glucose. The arginine:ADMA ratio showed an association (OR 0.976, $95 \%$ CI 0.963, 0.997, $P=0.025)$ and a diagnostic accuracy (area under the curve $0 \cdot 721,95 \%$ CI $0.560,0 \cdot 882, P=0 \cdot 016$ ) for hospital mortality, whereas the arginine or ADMA concentration alone or APACHE II-predicted mortality failed to do so. In conclusion, in shock patients, the imbalance of arginine and ADMA is related to circulatory failure, organ failure and disease severity, and predicts mortality. We propose a pathophysiological mechanism in shock: the imbalance of arginine and ADMA contributes to endothelial and cardiac dysfunction resulting in poor organ perfusion and organ failure, thereby increasing the risk of death.
\end{abstract}

Key words: Arginine: Asymmetric dimethylarginine: Shock: Circulation

Arginine, the sole NO precursor and a semi-essential amino acid, is thought to become essential in shock patients. Septic $^{(1)}$ and cardiogenic shock ${ }^{(2)}$ are characterised by low arginine and excessive NO levels. Several studies have tried to boost arginine levels in critically ill patients by supplementing this amino acid alone or in combination with other immunomodulating substances. Results of the studies are controversial. While some have shown beneficial or no effects $^{(3-5)}$, others have in fact suggested that arginine might increase the risk of mortality ${ }^{(6,7)}$. A possible negative effect of arginine might have been mediated by arginine-induced increased NO production by inducible NO synthase (NOS), which in turn could have led to detrimental systemic vasodilation ${ }^{(8)}$ or to increased formation of peroxynitrite due to concomitant oxyradical production inducing cellular damage ${ }^{(9)}$. On the other hand, arginine may have positive effects by enhancing NO-mediated microvascular vasodilatation facilitated by endothelial NOS, which is crucial for organ perfusion and coronary

Abbreviations: ADMA, asymmetric dimethylarginine; APACHE, Acute Physiology and Chronic Health Evaluation; AUC, area under the curve; ICU, intensive care unit; NICO, non-invasive cardiac output; NOS, nitric oxide synthase; SOFA, Sequential Organ Failure Assessment.

* Corresponding author: Professor P. A. M. van Leeuwen, fax +3120444 3620, email pam.vleeuwen@vumc.nl 
patency. Probably NO availability needs to be perfectly balanced $^{(10)}$

Quantitatively, arginine consumption by NOS is partly determined by the availability of NOS inhibitors, such as asymmetric dimethylarginine (ADMA) that facilitates vasoconstriction and deteriorates endothelial and cardiac function ${ }^{(11)}$. In critically ill patients, highly elevated levels of ADMA were observed, and high ADMA predicted mortality and correlated with organ severity ${ }^{(12)}$. The above findings suggest that NO availability might depend on the balance of a NOS substrate (arginine) and an inhibitor (ADMA). Indeed, in a recent study by our group, low arginine and high ADMA levels reduced cardiac output in rats ${ }^{(13)}$.

Based on previous studies, we hypothesise that an imbalance of arginine and ADMA contributes to poor organ perfusion in patients with shock. Therefore, we investigated the relationship between arginine, ADMA and their ratio at admission to the intensive care unit (ICU) and circulatory markers, disease severity, organ failure and mortality in critically ill shock patients.

\section{Materials and methods}

\section{Patients}

The present prospective cohort study was conducted in a twenty-bed closed format general ICU of a teaching hospital in the city of Amsterdam, The Netherlands. The study included adult patients with persistent septic or cardiogenic shock within $24 \mathrm{~h}$ after ICU admission, requiring mechanical ventilation. Other inclusion criteria were predicted ICU treatment and intention to treat for at least $5 \mathrm{~d}$. Exclusion criteria were active massive bleeding, pregnancy, HIV with less than fifty CD4 cells, haematologic malignancy, metastatic malignancy, Child $\mathrm{C}$ liver cirrhosis, hepatic coma and therapeutic hypothermia after cardiac arrest.

For inclusion into the study, shock was defined as persistent hypotension despite adequate fluid resuscitation and the need of dopamine at a dose of more than $6 \mu \mathrm{g} / \mathrm{kg}$ per min and any dose of additional noradrenaline in the presence of perfusion abnormalities, manifest by oliguria, reduced peripheral perfusion and organ dysfunction. Hypotension was defined as a systolic pressure $<90 \mathrm{mmHg}$; oliguria as a urinary output $<20 \mathrm{ml} / \mathrm{min}$ despite fluid infusion; reduced peripheral perfusion as $\Delta \mathrm{T}$ (difference between the core and peripheral temperature) $<4^{\circ} \mathrm{C}$, skin colour not pink, poor capillary refill; and organ dysfunction was defined according to the Sequential Organ Failure Assessment (SOFA) score ${ }^{(14)}$. Septic shock was defined as the form of acute circulatory shock occurring secondary to severe infection ${ }^{(15)}$. Cardiogenic shock is the form of circulatory shock occurring secondary to heart failure as evidenced by low cardiac output or ejection fraction accompanying cardiac disease (supported by, for example, echocardiography). To optimise the circulation, we used fluids in a mixture of crystalloids and colloids (Gelofusine ${ }^{\circledR}$; B. Braun, Melsungen, Germany) and dopamine as a first-line inotropic and vasopressor agent. Noradrenaline was added if higher doses of dopamine were needed and/or if the patient developed tachycardia. Enoximone was added when the cardiac index remained $<2.51 / \mathrm{kg}$ per $\mathrm{h}$ at a standard dose of $8 \mathrm{mg} / \mathrm{h}$. Nitroglycerin was used in patients with cardiogenic shock, cardiac ischaemia and/or persistent poor peripheral perfusion $^{(16)}$. Fluids were infused in amounts considered to be necessary to restore circulating volume, and to optimise cardiac output and peripheral circulation. Endpoints of the circulation treatment were $\Delta \mathrm{T}$, skin colour, capillary refill, blood pressure (target $>90 \mathrm{mmHg}$ ), central venous pressure, cardiac index (target $>2.51 / \mathrm{kg}$ per $\mathrm{min}$ ), pulse pressure variation (target $<10 \%$ if appropriate) and mixed venous oxygen saturation (target $>70 \mathrm{mmHg}$ if feasible).

The study was conducted according to the guidelines laid down in the Declaration of Helsinki and all procedures involving patients were approved by the medical ethics committee of the Onze Lieve Vrouwe Gasthuis. Written informed consent was obtained from all patients or their legal representative. Patients were followed until they died (non-survivors) or were discharged from the hospital (survivors).

\section{Physiological and laboratory parameters}

Blood samples were taken after the inclusion criteria were met in the first $24 \mathrm{~h}$ of ICU admission and were immediately placed on ice and centrifuged. Plasma was pipetted and immediately put in liquid $\mathrm{N}_{2}$ and stored at $-80^{\circ} \mathrm{C}$ before analysis. The concentrations of arginine and ADMA were measured by HPLC on a monolithic column as described previously ${ }^{(17,18)}$. Intra- and inter-assay $\mathrm{CV}$ for both arginine and ADMA were $<2$ and $<3 \%$, respectively. In addition, the arginine:ADMA ratio was calculated. Normal values for arginine and ADMA concentrations were $80(\mathrm{SD} \mathrm{20})^{(19)}$ and $0.497(\mathrm{SD} 0.063) \mu \mathrm{mol} / \mathrm{l}^{(17)}$, respectively.

Laboratory parameters indicating hepatic and renal function (bilirubin, alanine aminotransferase, creatinine (clearance) and urea), muscle degradation (creatine kinase) and acid-base physiology (bicarbonate, $\mathrm{pH}$ and lactate) were analysed by standard laboratory methods. Diagnosis of disseminated intravascular coagulation was done by calculation of the global coagulation test score ${ }^{(20)}$. At ICU admission, cardiac output was assessed invasively with the Swan Ganz catheter based on the thermodilution method, or non-invasively with cardiac output (NICO; Novametrix Medical Systems, Inc., Wallingford, CT, USA) that continuously measures pulmonary perfusion based on the Fick principle. NICO was preferred, especially in patients with sepsis, since it offers the benefit of continuous monitoring and avoids the risks of invasive monitoring. Cardiac index was calculated by dividing the cardiac output by the body surface area. Furthermore, severity of illness was scored using the Acute Physiology and Chronic Health Evaluation (APACHE) II system over the first $24 \mathrm{~h}$ of ICU admission ${ }^{(21)}$, and APACHE II-predicted mortality was used as a reference for the predictive values of arginine, ADMA and their ratio for mortality ${ }^{(22)}$. The SOFA score as defined by the Dutch National Intensive Care Evaluation (www.stichting-nice.nl) was measured daily ${ }^{(14)}$. 
Table 1. Patient characteristics and biochemical values

(Number, percentage, median values, interquartile ranges, mean values and standard deviations)

\begin{tabular}{cccr}
\hline & Mean & SD \\
\hline Demographics & & & \\
Sex & & & \\
Female & & & \\
$n$ & & 21 & \\
$\%$ & & 47.7 & \\
Male & & 23 & \\
$n$ & & 52.3 & \\
$\%$ & 65.7 & & 13.8 \\
Age (years) & 172.5 & & 8.9 \\
Height (cm) & 75.3 & & 17.4 \\
Weight (kg) & & &
\end{tabular}

Clinical assessment

ICU admission type

Medical

\%

Surgical

$n$

$\%$

ICU stay (d)

Median

IQR

ICU mortality

$n$

$\%$

Hospital mortality

$n$

APACHE II score

APACHE II-predicted mortality

SOFA score

Cardiac index $\left(1 /\right.$ min per $\left.\mathrm{m}^{2}\right)$

Shock

Septic

$n$
$\%$

$\%$

Cardiogenic

$n$

Site of infection (\%)

Lung

$n$
$\%$

$\%$

Abdomen

$n$

$\%$

Urogenital

$n$

$\%$

Other

$n$
$\%$

None (cardiogenic shock)

$n$

$\%$

Dose of vasoactive agents at day 1

Dopamine $(\mu \mathrm{g} / \mathrm{kg}$ per $\mathrm{min})$

Noradrenaline ( $\mu \mathrm{g} / \mathrm{kg}$ per $\mathrm{min}$ )

Nitroglycerin $(\mu \mathrm{g} / \mathrm{kg}$ per $\mathrm{min})$

Laboratory measurements

Bilirubin $(\mu \mathrm{mol} / \mathrm{l})$

ALAT (U/l)

Median

IQR

Creatinine $(\mu \mathrm{mol} / \mathrm{l})$

Median

IQR

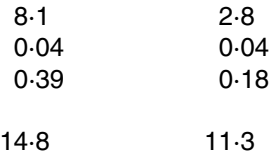

29.0

$15-67$

$124 \cdot 5$

$87 \cdot 3-167 \cdot 8$
Table 1. Continued

\begin{tabular}{|c|c|c|}
\hline & Mean & SD \\
\hline \multicolumn{3}{|c|}{ Creatinine clearance $(\mathrm{ml} / \mathrm{min})$} \\
\hline Median & \multirow{2}{*}{\multicolumn{2}{|c|}{$\begin{array}{c}52 \cdot 3 \\
32.2-67.2\end{array}$}} \\
\hline IQR & & \\
\hline \multicolumn{3}{|l|}{ Urea (mmol/l) } \\
\hline Median & \multirow{2}{*}{\multicolumn{2}{|c|}{$\begin{array}{c}9.4 \\
7.1-17.4\end{array}$}} \\
\hline IQR & & \\
\hline \multicolumn{3}{|l|}{ Creatine kinase (IU/I) } \\
\hline Median & \multirow{2}{*}{\multicolumn{2}{|c|}{$\begin{array}{c}99.5 \\
60.3-458.5\end{array}$}} \\
\hline IQR & & \\
\hline DIC & 2.8 & 1.2 \\
\hline Bicarbonate $(\mathrm{mmol} / \mathrm{l})$ & $18 \cdot 7$ & 5.9 \\
\hline \multicolumn{3}{|l|}{ Lactate $(\mathrm{mmo} / \mathrm{l})$} \\
\hline Median & \multirow{2}{*}{\multicolumn{2}{|c|}{$\begin{array}{c}3.6 \\
1.9-6.4\end{array}$}} \\
\hline IQR & & \\
\hline $\mathrm{pH}$ & $7 \cdot 3$ & 0.13 \\
\hline Glucose (mmol/l) & 9.5 & $5 \cdot 0$ \\
\hline $\operatorname{Arg}(\mu \mathrm{mol} / \mathrm{l})$ & $34 \cdot 6$ & $17 \cdot 9$ \\
\hline ADMA $(\mu \mathrm{mol} / \mathrm{l})$ & 0.46 & 0.18 \\
\hline Arg:ADMA & 83.2 & 42.5 \\
\hline
\end{tabular}

ICU, intensive care unit; IQR, interquartile ranges; APACHE, Acute Physiology and Chronic Health Evaluation; SOFA, Sequential Organ Failure Assessment; ALAT, alanine aminotransferase; DIC, disseminated intravascular coagulation; ADMA, asymmetric dimethylarginine.

\section{Statistical analysis}

Data are expressed as means and standard deviations in the case of normally distributed data and as medians and interquartile ranges when data are not normally distributed. Normality was tested by the Shapiro-Wilk normality test. Likewise, we used Pearson's correlation and Spearman's rank correlation coefficient to determine whether clinical and biochemical variables were significantly related to arginine, ADMA and their ratio. Predictors of mortality were studied by calculation of the OR in a logistic regression model with one variable (arginine, ADMA, arginine:ADMA ratio or APACHE II-predicted mortality). Our sample size did not permit a multiple regression analysis. Receiver-operating characteristic curves were estimated using the non-parametric method to further evaluate the association of arginine, ADMA, the arginine:ADMA ratio and APACHE II-predicted mortality with hospital mortality. The area under the curve (AUC) was calculated to determine the accuracy of arginine, ADMA, the arginine:ADMA ratio and APACHE II-predicted mortality as predictors of hospital mortality. The further the curve lies above the reference line and the higher the AUC, the more accurate is the test. Coordinates of the curve were examined across the full range of potential arginine:ADMA cut-off values in an attempt to select an optimal arginine:ADMA cut-off value that properly balanced the needs of sensitivity and specificity. Furthermore, positive and negative predictive cut-off values of arginine:ADMA were calculated. The $\chi^{2}$ test was used to analyse the difference in mortality between patients with an arginine:ADMA ratio below the cut-off value and those with an arginine:ADMA ratio above the cut-off value. A $P$ value of $<0.05$ (two-tailed) was considered to be statistically significant. Statistical analysis was performed with SPSS 15.0 for Windows (SPSS Inc., Chicago, IL, USA). 


\section{Results}

\section{Patients}

Patient characteristics and biochemical values are presented in Table 1. Of the forty-four shock patients, twenty-seven had septic shock and seventeen had cardiogenic shock. At admission, the mean APACHE II score was 26.2 (SD 7.4) and the SOFA score was $10 \cdot 0$ (SD 3.0). Mean levels of arginine and ADMA were 34.6 (SD 17.9) and 0.46 (SD 0.18) $\mu \mathrm{mol} / \mathrm{l}$, respectively, and the mean arginine:ADMA ratio was 83.2 (SD 42.5). The levels of arginine, ADMA, the arginine:ADMA ratio, the APACHE II and SOFA score and the cardiac index did not differ significantly between septic and cardiogenic shock patients (arginine: 32.2 (SD 15.9) and 38.4 (SD 20.7), $P=0 \cdot 27$; ADMA: 0.43 (SD 0.16 ) and 0.50 (SD 0.20), $P=0.19$; arginine:ADMA: 79.9 (SD 33.7) and 88.4 (SD 54.4), $P=0.57$; APACHE II score: 26.5 (SD 7.7) and 25.6 (SD 7.2), $P=0.69$; SOFA score: 9.9 (SD 3.2) and 10.3 (SD 1.7), $P=0.89$; cardiac index: $2 \cdot 5(\mathrm{SD} 1 \cdot 1)$ and $2 \cdot 0(\mathrm{SD} 1 \cdot 0), P=0 \cdot 15)$.

\section{Arginine, asymmetric dimethylarginine and their ratio in shock patients}

Arginine correlated negatively with ICU length of stay, APACHE II-predicted mortality, SOFA score and urea. ADMA (tended to) correlate(d) positively with alanine aminotransferase, urea and glucose, and negatively with $\mathrm{pH}$. The arginine:ADMA ratio correlated with the markers of circulation (cardiac index (Fig. 1(a)), bicarbonate (Fig. 1(b)), lactate
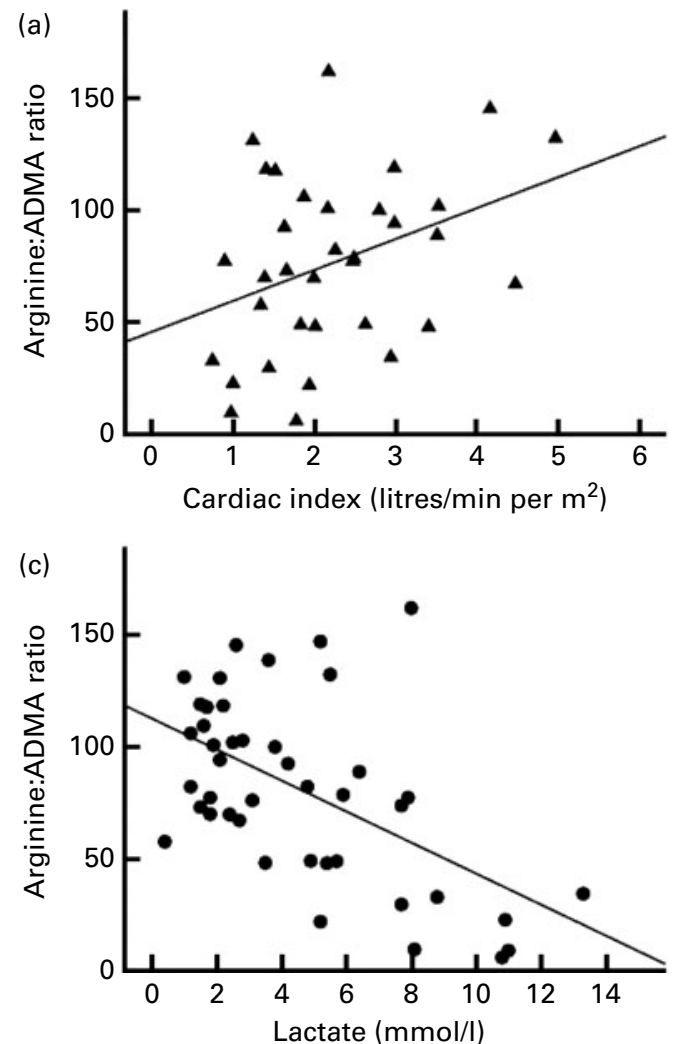

(Fig. 1(c)) and pH (Fig. 1(d))), and negatively with the APACHE II and SOFA score (Table 2). The arginine:ADMA ratio also correlated positively with creatine kinase and glucose (Table 2).

\section{Predictors of hospital mortality}

In a logistic regression model, the arginine:ADMA ratio predicted hospital mortality (OR 0.980, 95\% CI 0.963, 0.997, $P=0.025)$, while arginine, ADMA and APACHE II-predicted mortality were not significantly related to hospital mortality (OR 0.976, 95\% CI 0.940, 1.013, P=0.205; OR 40.9, $95 \% \mathrm{CI}$ 0.867, 1930, $P=0.059$; OR 3.47, 95\% CI 0.267, 45.2, $P=0.34$, respectively), although the relationship with ADMA tended to significance (Table 3). The receiver-operating characteristic curves in Fig. 2 reveal that the arginine:ADMA ratio (AUC: $0.721,95 \%$ CI $0.560,0.882, P=0.016$; Fig. 2(c)) provides better diagnostic accuracy to predict mortality compared with arginine (AUC: 0.621, 95\% CI 0.443, 0.798, $P=0.188$; Fig. 2(a)), ADMA (AUC: 0.706, 95\% CI 0.533, 0.880, $P=0.024$; Fig. 2(b)) and APACHE II-predicted mortality (AUC: $0.583,95 \%$ CI $0.401,0.765, P=0.367$; Fig. 2(d)). The optimal arginine:ADMA cut-off value was 93.4 (sensitivity: 0.875 , specificity: 0.571 ), with a positive predictive value of 0.54 (95\% CI $0.36,0.71$ ) and a negative predictive value of 0.89 (95\% CI 0.67, 0.97; Table 4). Hospital mortality was significantly higher in patients with an arginine:ADMA ratio below the cut-off value compared with patients above the cut-off value (14:24 v. 2:18, $\chi^{2}$ test, $\left.P=0 \cdot 004\right)$.
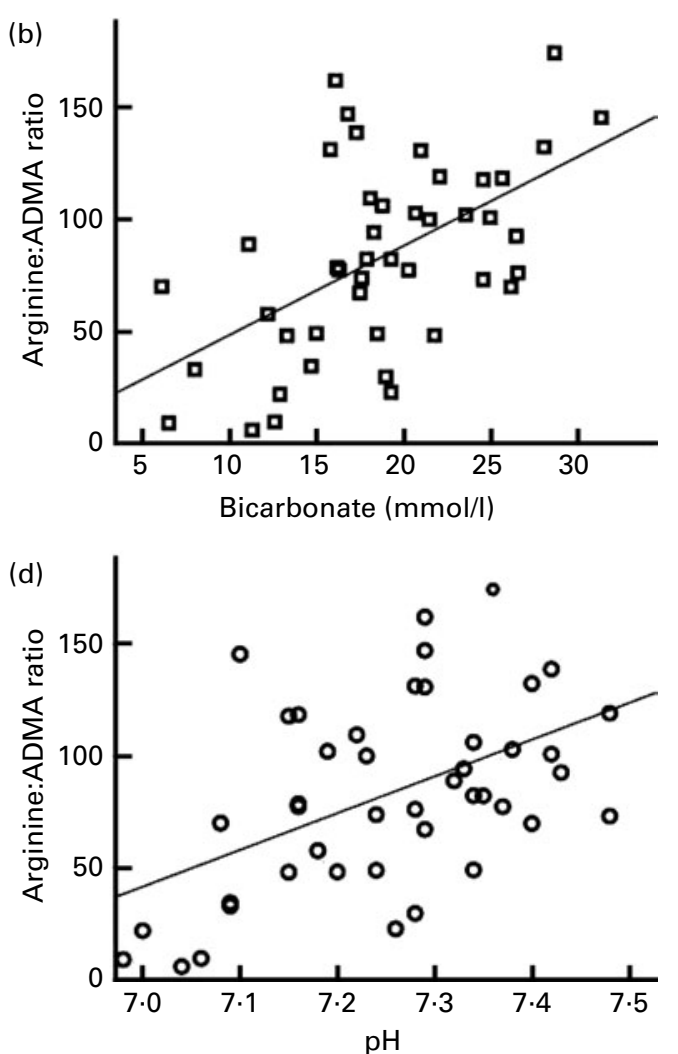

Fig. 1. Arginine:asymmetric dimethylarginine (ADMA) ratio correlates with the markers of circulation. Cardiac index: $r^{2} 0 \cdot 132$; bicarbonate: $r^{2} 0.305$; lactate: $r^{2}$ 0.218; $\mathrm{pH}: r^{2} 0.248$. 
Table 2. Correlations of arginine, asymmetric dimethylarginine (ADMA) and the arginine:ADMA ratio and clinical and biochemical variables

\begin{tabular}{|c|c|c|c|c|c|c|}
\hline & \multicolumn{2}{|c|}{ Arg } & \multicolumn{2}{|c|}{ ADMA } & \multicolumn{2}{|c|}{ Arg:ADMA } \\
\hline & $r$ & $P$ & $r$ & $P$ & $r$ & $P$ \\
\hline ICU stay* & -0.412 & 0.027 & -0.040 & 0.794 & -0.070 & 0.652 \\
\hline APACHE II score & -0.198 & 0.302 & 0.086 & 0.581 & -0.314 & 0.038 \\
\hline APACHE II-predicted mortality & -0.386 & 0.010 & -0.062 & 0.688 & -0.256 & 0.093 \\
\hline SOFA score & -0.391 & 0.009 & 0.160 & 0.301 & -0.362 & 0.016 \\
\hline Cardiac index & 0.176 & 0.320 & -0.279 & 0.110 & 0.364 & 0.034 \\
\hline Bilirubin & -0.004 & 0.978 & 0.261 & 0.087 & -0.154 & 0.317 \\
\hline $\mathrm{ALAT}^{*}$ & -0.126 & 0.516 & 0.303 & 0.045 & -0.790 & 0.612 \\
\hline Creatinine* $^{*}$ & -0.011 & 0.944 & -0.027 & 0.863 & -0.021 & 0.891 \\
\hline Creatinine clearance ${ }^{*}$ & 0.044 & 0.775 & 0.005 & 0.974 & $0 \cdot 123$ & 0.427 \\
\hline Urea* & -0.436 & 0.018 & 0.285 & 0.061 & -0.095 & 0.540 \\
\hline Creatine kinase ${ }^{*}$ & -0.319 & 0.092 & -0.048 & 0.755 & 0.371 & 0.013 \\
\hline Bicarbonate & 0.156 & 0.420 & -0.176 & 0.253 & 0.552 & $<0.001$ \\
\hline $\mathrm{pH}$ & 0.125 & 0.518 & -0.380 & 0.011 & 0.498 & 0.001 \\
\hline Lactate* $^{*}$ & 0.070 & 0.717 & 0.267 & 0.084 & -0.467 & 0.002 \\
\hline $\mathrm{DIC}$ & -0.097 & 0.625 & 0.245 & 0.118 & -0.409 & 0.007 \\
\hline Glucose & -0.220 & 0.151 & 0.395 & 0.008 & 0.337 & 0.025 \\
\hline
\end{tabular}

\section{Discussion}

The present observational cohort study in critically ill patients with shock shows that an imbalance of arginine and ADMA at ICU admission is associated with circulatory failure, severity of disease and organ failure, and also predicts mortality. The imbalance of arginine and ADMA was mainly the result of arginine depletion because ADMA levels were within the normal range. Furthermore, the arginine:ADMA ratio was related to all markers of circulation while arginine and ADMA separately were not. In addition, the ratio showed a stronger association and better diagnostic accuracy for hospital mortality in the present study, whereas the arginine or ADMA concentration alone failed to do so. It therefore seems that the balance of arginine and ADMA might be important for proper microand macrocirculation and not their individual concentrations. When arginine is depleted, a normal ADMA concentration may be relatively high. In the present shock patients, this imbalance between the NO substrate (arginine) and the inhibitor (ADMA) may result in poor organ perfusion.

Since poor organ perfusion is the main cause of organ failure, and both arginine and ADMA influence endothelial and cardiac function ${ }^{(11)}$, the present observations support the role of an imbalance between the NO substrate and the inhibitor in the pathophysiological mechanism of circulatory failure, organ failure and mortality in patients with shock. We propose a mechanism in which a combination of reduced flow to the organs and diminished microcirculation within these organs as a result of an imbalance between arginine and ADMA will end up in organ failure and an increased risk of death in shock patients. The associations with mortality and disease severity in septic patients with low arginine:ADMA ratios, as reported in the literature ${ }^{(23,24)}$ and the associations found in the present study, support this mechanism. The magnitude of the OR of 0.98 can be illustrated by an example: for a patient with an arginine:ADMA ratio of 80 , the odds of dying are $1.50\left(1 /\left(0 \cdot 98^{20}\right)\right)$ times higher than for a patient with an arginine:ADMA ratio of 100 .

The present results are in accordance with an experimental study done by our group in which a low arginine:ADMA ratio reduced cardiac output and diminished flow in the microcirculation of major organs in animals ${ }^{(13)}$. In addition, exogenous arginine increased coronary blood flow and restored perfusion in the ischaemic areas in the endotoxin-treated heart ${ }^{(25)}$, and increased cardiac output in sepsis ${ }^{(26,27)}$. On the other hand, safety of arginine administration remains controversial because two studies have shown increased mortality rates with supplementation of this amino acid in sepsis ${ }^{(6,7)}$. However, both studies have used immunonutrition with a low-arginine formula, which makes it difficult to attribute this effect to a single nutrient. Furthermore, the strength of the design of one of these studies ${ }^{(6)}$ can be doubted as different routes of feeding were used and the statistical significance was quite thin since one more deceased subject would have made the difference non-significant. The influence of ADMA has been supported by studies in which administration of NOS inhibitors reduced cardiac output in healthy volunteers ${ }^{(28)}$ and in septic shock patients ${ }^{(29)}$, and reduced coronary flow $^{(25)}$ and induced local ischaemia ${ }^{(25)}$ in endotoxaemic rats.

Table 3. Risk factors for hospital mortality

(Odd ratios and $95 \%$ confidence intervals)

\begin{tabular}{lcll}
\hline & OR & \multicolumn{1}{c}{$95 \% \mathrm{Cl}$} & \multicolumn{1}{c}{$P$} \\
\hline Arg & 0.976 & $0.940,1.013$ & 0.205 \\
ADMA & 40.9 & $0.867,1930$ & 0.059 \\
Arg:ADMA & 0.980 & $0.963,0.997$ & 0.025 \\
APACHE II-predicted mortality & 3.47 & $0.267,45.2$ & 0.34 \\
\hline
\end{tabular}

ADMA, asymmetric dimethylarginine; APACHE, Acute Physiology and Chronic Health Evaluation. 

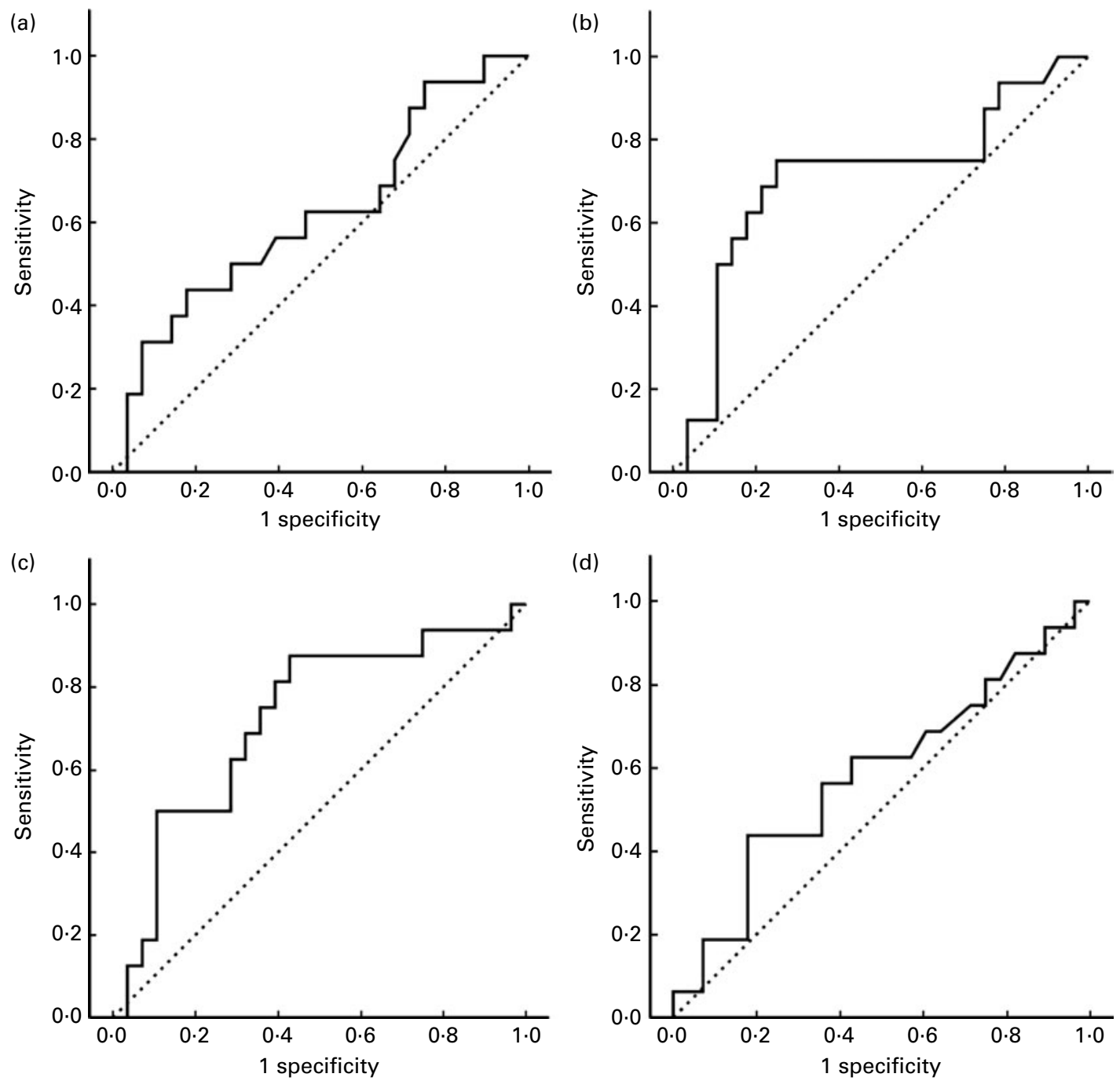

Fig. 2. Receiver-operating characteristic curves of (a) arginine, (b) asymmetric dimethylarginine (ADMA), (c) arginine:ADMA ratio and (d) Acute Physiology and Chronic Health Evaluation II-predicted mortality.

Furthermore, a study with cardiogenic shock patients has suggested that ADMA can regulate flow to the lung as the NOS inhibitor was associated with pulmonary capillary wedge pressure and with both systolic and diastolic pulmonary artery pressures ${ }^{(2)}$. Nevertheless, NOS inhibitors have been proposed as a treatment for the overproduction of $\mathrm{NO}$ in sepsis ${ }^{(29)}$. The hypothesis underlying the use of NOS inhibitors is that the increased production of NO by inducible NO synthase in sepsis contributes to hypotension and multiple organ dysfunction ${ }^{(30)}$. However, results of these experiments are conflicting ${ }^{(26,29,31)}$. Moreover, a non-selective NOS inhibitor increased mortality rate of septic shock patients, which was associated with decreased cardiac output and heart failure $^{(31)}$. Overcorrection of vascular tone by the non-selective NOS inhibitor might have hampered organ perfusion, resulting in myocardial and vascular dysfunction.

The observed low arginine:ADMA ratio in patients with high severity of disease and organ failure, and low cardiac index may be the result of decreased production or increased degradation of arginine and/or increased production or decreased clearance of ADMA, or both of these. In our patients, ADMA levels $(0.44$ (SD 0.15$) \mu \mathrm{mol} / \mathrm{l})$ at ICU admission were not elevated compared with normal values $(0.5 \mu \mathrm{mol} / \mathrm{l})^{(17)}$, while arginine levels were decreased (32.2 (SD 16.6) compared with $80 \mu \mathrm{mol} / 1$ in healthy subjects $\left.{ }^{(19)}\right)$. Similar results were

Table 4. Predictive values of the arginine:asymmetric dimethylarginine (ADMA) ratio for hospital mortality

(Mean values and $95 \%$ confidence intervals)

\begin{tabular}{lccc}
\hline & \multicolumn{3}{c}{ Arg:ADMA ratio } \\
\cline { 2 - 4 } & $<93.4$ & $\geq 93.4$ & $P^{\star}$ \\
\hline Survivors & 12 & 16 & 0.004 \\
Non-survivors & 14 & 2 & \\
Positive predictive value & & & \\
$\quad$ Mean & 0.54 & & \\
$\quad 95 \% \mathrm{Cl}$ & $0.36,0.71$ & & \\
Negative predictive value & & 0.89 & \\
$\quad$ Mean & & $0.67,0.97$ \\
$95 \% \mathrm{Cl}$ & & \\
\hline
\end{tabular}


found in a study by Mittermayer et al. ${ }^{(32)}$ in healthy males after the injection of Escherichia coli endotoxin: arginine concentration decreased while ADMA levels were not affected, resulting in a decline in the arginine:ADMA ratio. These observations suggest that the effect of the arginine:ADMA ratio on clinical outcome can mainly be due to low arginine levels. Arginine depletion in shock is probably the result of diminished production of arginine $e^{(33)}$ or its precursor citrulline ${ }^{(1)}$.

The limitations of the present study need to be addressed. Unfortunately, we could not measure NO or its oxidation products nitrite and nitrate. NO is highly reactive and has a short half-life $(<0 \cdot 1 \mathrm{~s} \text { in the human circulation })^{(34)}$. Furthermore, plasma levels of NO oxidation products are influenced by several factors such as endogenous NO synthesis, dietary intake and excretion, and clinical and therapeutic interventions. Therefore, the production of $\mathrm{NO}$, nitrite and nitrate may not be reliably assessed in shock patients. Second, cardiac output was not uniformly measured, because we used both the pulmonary artery catheter and NICO for cardiac output measurement. This choice is based on the absence of clinical evidence that the use of a pulmonary artery catheter reduces morbidity or mortality and that a continuous measurement, as provided by NICO, improves optimisation of the circulation at the bedside and reduces the risk of infection in septic patients $^{(35)}$. However, cardiac output is only one of the markers of circulation. We also found a significant relationship with other markers of circulation such as disseminated intravascular coagulation score, bicarbonate, lactate and metabolic acidosis. Finally, because of the small sample size and the observational design of the present study, it is not possible to draw conclusions about the cause-effect relationship of the present results.

In conclusion, the main finding of the present observational study is that an imbalance of arginine and ADMA at ICU admission is associated with circulatory failure, organ failure and mortality in patients with septic or cardiogenic shock. In the present study, the imbalance was caused by arginine depletion, while ADMA concentrations were normal. However, the ratio was related to the markers of circulation and outcome, while arginine and ADMA levels alone were not. These results support the present hypothesis that an imbalance of arginine and ADMA, being the substrate and the inhibitor of NO synthase, respectively, might contribute to endothelial and cardiac dysfunction with subsequent poor organ perfusion and organ failure, thereby increasing the risk of mortality. Future studies should focus on the role of arginine supplementation or ADMA removal in a specific subgroup of early shock with persisting circulatory failure.

\section{Acknowledgements}

M. V. was supported by the Egbers Foundation. M. V. and M. A. R. V. contributed equally to this study and share first authorship. They substantially performed the statistical analysis and drafted the manuscript. H. M. O.-v. S. conceived the design and execution of the study, contributed to the interpretation of the data and the writing of the manuscript, and was responsible for all parts of the research. T. T. carried out the sample analyses. P. J. K. helped performing and interpreting the statistical analysis. M. C. R., A. P. J. H., W. W., B. A. J. M. d. M. and P. A. M. V. L. critically analysed and interpreted the data and helped writing the manuscript. All authors read and approved the final manuscript. The authors have no conflicts of interest to declare.

\section{References}

1. Luiking YC, Poeze M, Ramsay G, et al. (2009) Reduced citrulline production in sepsis is related to diminished de novo arginine and nitric oxide production. Am J Clin Nutr 89, 142-152.

2. Nicholls SJ, Wang Z, Koeth R, et al. (2007) Metabolic profiling of arginine and nitric oxide pathways predicts hemodynamic abnormalities and mortality in patients with cardiogenic shock after acute myocardial infarction. Circulation 116, 2315-2324.

3. Bower RH, Cerra FB, Bershadsky B, et al. (1995) Early enteral administration of a formula (Impact) supplemented with arginine, nucleotides, and fish oil in intensive care unit patients: results of a multicenter, prospective, randomized, clinical trial. Crit Care Med 23, 436-449.

4. Caparros T, Lopez J \& Grau T (2001) Early enteral nutrition in critically ill patients with a high-protein diet enriched with arginine, fiber, and antioxidants compared with a standard high-protein diet. The effect on nosocomial infections and outcome. JPEN J Parenter Enteral Nutr 25, 299-308.

5. Tepaske R, Velthuis H, Oudemans-van Straaten HM, et al. (2001) Effect of preoperative oral immune-enhancing nutritional supplement on patients at high risk of infection after cardiac surgery: a randomised placebo-controlled trial. Lancet 358, 696-701.

6. Bertolini G, Iapichino G, Radrizzani D, et al. (2003) Early enteral immunonutrition in patients with severe sepsis: results of an interim analysis of a randomized multicentre clinical trial. Intensive Care Med 29, 834-840.

7. Dent D, Heyland D \& Levy H (2003) Immunonutrition may increase mortality in critically ill patients with pneumonia: results of a randomized trial. Crit Care Med 30, A17.

8. Suchner U, Heyland DK \& Peter K (2002) Immune-modulatory actions of arginine in the critically ill. Br J Nutr $\mathbf{8 7}$, Suppl. 1, S121-S132.

9. Xia Y, Dawson VL, Dawson TM, et al. (1996) Nitric oxide synthase generates superoxide and nitric oxide in argininedepleted cells leading to peroxynitrite-mediated cellular injury. Proc Natl Acad Sci U S A 93, 6770-6774.

10. Preiser JC, Luiking Y \& Deutz N (2011) Arginine and sepsis: a question of the right balance? Crit Care Med 39, 1569-1570.

11. Visser M, Paulus WJ, Vermeulen MA, et al. (2010) The role of asymmetric dimethylarginine and arginine in the failing heart and its vasculature. Eur J Heart Fail 12, 1274-1281.

12. Nijveldt RJ, Teerlink T, van der Hoven B, et al. (2003) Asymmetrical dimethylarginine (ADMA) in critically ill patients: high plasma ADMA concentration is an independent risk factor of ICU mortality. Clin Nutr 22, 23-30.

13. Richir MC, van Lambalgen AA, Teerlink T, et al. (2009) Low arginine/asymmetric dimethylarginine ratio deteriorates systemic hemodynamics and organ blood flow in a rat model. Crit Care Med 37, 2010-2017.

14. Vincent JL, Moreno R, Takala J, et al. (1996) The SOFA (Sepsis-related Organ Failure Assessment) score to describe organ dysfunction/failure. On behalf of the Working Group 
on Sepsis-Related Problems of the European Society of Intensive Care Medicine. Intensive Care Med 22, 707-710.

15. American College of Chest Physicians/Society of Critical Care Medicine Consensus Conference: definitions for sepsis and organ failure and guidelines for the use of innovative therapies in sepsis. (1992) Crit Care Med 20, 864-874.

16. Spronk PE, Ince C, Gardien MJ, et al. (2002) Nitroglycerin in septic shock after intravascular volume resuscitation. Lancet 360, 1395-1396.

17. Teerlink T (2007) HPLC analysis of ADMA and other methylated L-arginine analogs in biological fluids. $J$ Chromatogr $B$ Analyt Technol Biomed Life Sci 851, 21-29.

18. de Jong S \& Teerlink T (2006) Analysis of asymmetric dimethylarginine in plasma by HPLC using a monolithic column. Anal Biochem 353, 287-289.

19. Cynober LA (2002) Plasma amino acid levels with a note on membrane transport: characteristics, regulation, and metabolic significance. Nutrition 18, 761-766.

20. Taylor FB Jr, Toh CH, Hoots WK, et al. (2001) Towards definition, clinical and laboratory criteria, and a scoring system for disseminated intravascular coagulation. Thromb Haemost 86, 1327-1330.

21. Knaus WA, Draper EA, Wagner DP, et al. (1985) APACHE II: a severity of disease classification system. Crit Care Med 13, 818-829.

22. Goldhill DR \& Withington PS (1996) Mortality predicted by APACHE II. The effect of changes in physiological values and post-ICU hospital mortality. Anaesthesia 51, 719-723.

23. Gough MS, Morgan MA, Mack CM, et al. (2011) The ratio of arginine to dimethylarginines is reduced and predicts outcomes in patients with severe sepsis. Crit Care Med 39, 1351-1358.

24. Davis JS, Darcy CJ, Yeo TW, et al. (2011) Asymmetric dimethylarginine, endothelial nitric oxide bioavailability and mortality in sepsis. PLoS One 6, e17260.

25. Avontuur JA, Bruining HA \& Ince C (1995) Inhibition of nitric oxide synthesis causes myocardial ischemia in endotoxemic rats. Circ Res 76, 418-425.
26. Lorente JA, Landin L, De PR, et al. (1993) L-Arginine pathway in the sepsis syndrome. Crit Care Med 21, 1287-1295.

27. Pastor C, Teisseire B, Vicaut E, et al. (1994) Effects of $\mathrm{L}$-arginine and L-nitro-arginine treatment on blood pressure and cardiac output in a rabbit endotoxin shock model. Crit Care Med 22, 465-469.

28. Achan V, Broadhead M, Malaki M, et al. (2003) Asymmetric dimethylarginine causes hypertension and cardiac dysfunction in humans and is actively metabolized by dimethylarginine dimethylaminohydrolase. Arterioscler Thromb Vasc Biol 23 , $1455-1459$.

29. Petros A, Bennett D \& Vallance P (1991) Effect of nitric oxide synthase inhibitors on hypotension in patients with septic shock. Lancet 338, 1557-1558.

30. Nijveldt RJ, Teerlink T \& van Leeuwen PA (2003) The asymmetrical dimethylarginine (ADMA)-multiple organ failure hypothesis. Clin Nutr 22, 99-104.

31. Lopez A, Lorente JA, Steingrub J, et al. (2004) Multiplecenter, randomized, placebo-controlled, double-blind study of the nitric oxide synthase inhibitor 546C88: effect on survival in patients with septic shock. Crit Care Med 32, 21-30.

32. Mittermayer F, Namiranian K, Pleiner J, et al. (2004) Acute Escherichia coli endotoxaemia decreases the plasma L-arginine/asymmetrical dimethylarginine ratio in humans. Clin Sci (Lond) 106, 577-581.

33. Villalpando S, Gopal J, Balasubramanyam A, et al. (2006) In vivo arginine production and intravascular nitric oxide synthesis in hypotensive sepsis. Am J Clin Nutr 84, 197-203.

34. Baylis C \& Vallance P (1998) Measurement of nitrite and nitrate levels in plasma and urine - what does this measure tell us about the activity of the endogenous nitric oxide system? Curr Opin Nephrol Hypertens 7, 59-62.

35. Shah MR, Hasselblad V, Stevenson LW, et al. (2005) Impact of the pulmonary artery catheter in critically ill patients: meta-analysis of randomized clinical trials. JAMA $\mathbf{2 9 4}$ 1664-1670. 